\title{
EL PROFESIONAL DEL NEUROMARKETING EN EL SECTOR AUDIOVISUAL ESPAÑOL
}

\section{The neuromarketing professional in the Spanish audiovisual sector}

\author{
Verónica Crespo-Pereira, Valentín-Alejandro Martínez-Fernández y Pilar \\ García-Soidán
}

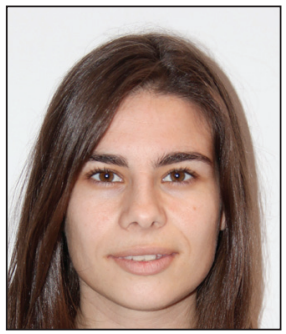

Verónica Crespo-Pereira es licenciada en publicidad y relaciones públicas por la Universidad de Vigo y máster en producción y gestión audiovisual por la Universidad de A Coruña. Su actividad profesional se ha desarrollado en el sector audiovisual en los departamentos de producción y dirección para series de ficción y programas. Trabaja como investigadora en la Universidad de Vigo donde lleva a cabo su tesis doctoral.

http://orcid.org/0000-0001-7373-7204

Universidad de Vigo, Facultad de Ciencias Sociales y de la Comunicación Campus A Xunqueira, s/n. 36005 Pontevedra, España veronicacrespopereira@gmail.com

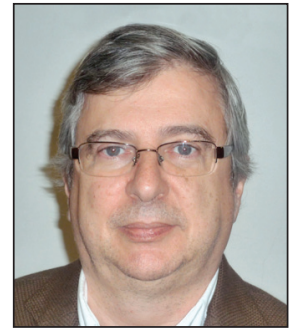

Valentín-Alejandro Martínez-Fernández es licenciado en ciencias de la información por la Universidad Complutense de Madrid (UCM). Máster MBA en dirección y administración de empresas por la Universidad de A Coruña (UDC). Doctor en ciencias de la información por la UCM y profesor titular de universidad en la Facultad de Ciencias de la Comunicación y en la Facultad de Economía y Empresa de la UDC. Ha sido director del diario El ideal gallego así como directivo en otras empresas informativas. Es autor de artículos sobre medios de comunicación y estrategias de comunicación. Es miembro del Proyecto Prometeo de la Secretaría de Educación Superior, Ciencia, Tecnología e Innovación de la República de Ecuador. Prometeo-CEB-008-2015. http://orcid.org/0000-0003-0069-675X

Universidad de A Coruña, Facultad de Economía y Empresa Campus Elviña, s/n. 15071, A Coruña, España valejand@udc.es

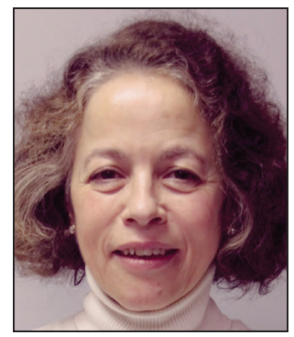

Pilar García-Soidán es licenciada y doctora en matemáticas por la Universidad de Santiago de Compostela. Desarrolla su actividad docente en la Universidad de Vigo, como catedrática de escuela universitaria del área de estadística e investigación operativa. Actualmente es la secretaria de la Comisión Académica del Doctorado en Comunicación. Su trayectoria profesional ha sido reconocida con tres sexenios de investigación. http://orcid.org/0000-0003-4542-6630

Universidad de Vigo, Facultad de Ciencias Sociales y de la Comunicación Campus A Xunqueira, s/n. 36005 Pontevedra, España pgarcia@uvigo.es

\section{Resumen}

La aparición de nuevos modelos de negocio digitales y su incidencia en el consumo de productos audiovisuales aboca a optimizar las decisiones y redefinir constantemente las estrategias de los grupos de comunicación tradicionales. Como respuesta a este nuevo escenario, en los últimos años algunas significativas empresas del sector han comenzado a incorporar novedosos avances metodológicos en su investigación de mercados, con el fin de dar una respuesta más eficiente a las demandas de las audiencias. La introducción del neuromarketing es una tendencia que, aun siendo incipiente, cobra cada vez más presencia en España. Con el fin de delimitar su alcance y proyección aplicados al sector audiovisual español, se ha llevado a cabo una investigación en la cual se analiza su grado de implantación y la nueva figura del profesional especializado en su gestión, al tiempo de definir prácticas, retos y desarrollo futuro.

\section{Palabras clave}

Neuromarketing; Profesional del neuromarketing; Industria audiovisual; Investigación de mercados. 


\begin{abstract}
The emergence of new digital business models and its subsequent impact on the consumption of audiovisual products has led to more optimized decisions and the constant refinement of the strategies of traditional media groups. In the last several years some major audiovisual companies have begun to incorporate novel methodological developments into their market research in order to give a more efficient response to audience demands. The introduction of neuromarketing is a trend that, although incipient, is becoming more established in Spain. In order to define the scope and impact of neuromarketing in its application to the Spanish audiovisual sector, this study aims to analyze both the implementation and the new professional who manages it, leading to a guide of best practices, challenges, and future development.
\end{abstract}

\title{
Keywords
}

Neuromarketing; Neuromarketing professional; Entertainment industry; Consumer research.

Crespo-Pereira, Verónica; Martínez-Fernández, Valentín-Alejandro; García-Soidán, Pilar (2016). “El profesional del neuromarketing en el sector audiovisual español". El profesional de la información, v. 25, n. 2, pp. 209-216.

http://dx.doi.org/10.3145/epi.2016.mar.07

\section{El neuromarketing en el sector audiovisual}

Desde los años noventa del pasado siglo, llamados por algunos "la década del cerebro", asistimos a la incorporación de conocimientos y técnicas de las neurociencias al campo del marketing con el objetivo de incrementar la efectividad de las acciones comerciales (Ariely; Berns, 2010; Braidot, 2009) que han dado lugar al denominado neuromarketing. Éste se puede definir como "la aplicación de técnicas de neuroimagen para entender la conducta humana en relación con los mercados e intercambios mercantiles" (Lee; Broderick; Chamberlain, 2007, p. 200).

A pesar de que el neuromarketing se encuentra en una etapa inicial (Hubert; Kenning, 2008), la introducción de metodologías, procedimientos y herramientas propias de la neurociencia como método complementario a las técnicas tradicionales del marketing, podría responder a las necesidades actuales de una industria audiovisual en pleno proceso de evolución que busca mejorar su competitividad y, en definitiva, el ROI (Mullen; Noble, 2007).

La interpretación de los resultados y su implementación en las empresas son algunas de las barreras a las que se enfrenta el neuromarketing

La aparición de plataformas digitales y nuevas formas de consumo audiovisual imponen cambios en el sistema publicitario y productivo conocido hasta ahora. Los retos a los que se enfrentan hoy las grandes empresas mediáticas tradicionales, hacen necesario investigar sobre fórmulas que permitan mantener y mejorar su posición en el mercado. En este sentido las industrias culturales, y especialmente las audiovisuales, están muy interesadas en la neurociencia (Ariely; Berns, 2010).

Empresas como el conglomerado mediático Viacom (Tiltman, 2005), Cartoon Network, ESPN o The Weather Channel (Babu; Vidyasagar, 2012) ya utilizan con distintos propósitos técnicas de neuromarketing en sus investigaciones de mercado. Las posibilidades que ofrecen estos estudios son múltiples:
- test de contenidos audiovisuales para acercarse más al gusto de la audiencia (Hammou; Galib; Melloul, 2013); - pruebas para la efectividad promocional de productos del sector cinematográfico y televisivo (Mullen; Noble, 2007);

- estudios para la mejora de los bloques publicitarios realizados por cadenas como Warner Bros (Treutler; Levine; Marci, 2010).

Un interesante estudio de Pop, Dabija y lorga (2014), realizado entre empresas de la asociación Nmsba (Neuromarketing Science \& Business Association), evidencia que los sectores que más solicitan servicios de neuromarketing son publicidad, diseño de productos y entretenimiento.

\section{La investigación neurocientífica en el sector audiovisual es de aplicación en contenidos, promoción de producciones y bloques publicitarios}

La citada Nmsba es una asociación internacional de empresas con intereses profesionales en neuromarketing. Su principal cometido es la divulgación de nuevos conocimientos, la protección de los intereses de la disciplina y la creación de una sólida red de contactos. Para formar parte de ella es necesario aceptar su código ético y abonar una cuota anual.

Para conocer la introducción de los servicios de neuromarketing en la industria del entretenimiento se han analizado las webs de los 41 países miembros de la Nmsba. De un total de 104 webs ( 5 no se han podido incluir al detectarse fallos técnicos), en 19 (18\%) se han encontrado referencias a servicios de neuromarketing para la industria del entretenimiento, que se recogen en la tabla 1. El país donde tienen su sede la mayoría de estas compañías es Estados Unidos con 6 empresas, seguido de Reino Unido con 2.

Existe una tendencia creciente a la colaboración entre universidades y grandes compañías mediáticas. Es el caso del proyecto Beyond:30, creado en 2005, que reúne a las mayores empresas de comunicación y entretenimiento del mundo, agencias, anunciantes y comunidad académica, con el ob- 
Tabla 1. Empresas de Nmsba con servicios a la industria del entretenimiento

\begin{tabular}{|c|c|}
\hline Empresa & Actividad en el campo del entretenimiento \\
\hline $\begin{array}{l}\text { Neurensics } \\
\text { Neuromarketing Research } \\
\text { (Argentina, Alemania y } \\
\text { Holanda) }\end{array}$ & $\begin{array}{l}\text { Estudios de posicionamiento de marca, entre otros. Entre sus clientes se encuentran Discovery Channel y National } \\
\text { Geographic. }\end{array}$ \\
\hline True Impact (Canadá) & Servicios a productoras de películas, documentales y programas de televisión. Tiene como partner a Neurons Inc. \\
\hline Mindmetriks (Colombia) & Usabilidad y experiencia del usuario en el campo de los videojuegos. \\
\hline $\begin{array}{l}\text { Brain Intelligence. } \\
\text { Neuro-Consultancy Ltd. } \\
\text { (China) }\end{array}$ & Se dirije a la industria del videojuego. Uno de sus clientes es Tencent \\
\hline Neurons Inc. (Dinamarca) & $\begin{array}{l}\text { Proporciona tres tipos de servicios: neuromarketing, neurocinematics y neurogaming, en clara alusión a la industria } \\
\text { audiovisual y del videojuego. Entre sus clientes dicen encontrarse } N B C \text {, Universal y } D R \text {. }\end{array}$ \\
\hline Innerscope Research (EUA) & En su cartera de clientes se encuentran empresas del sector de la comunicación como Fox Sports y Turner Broadcasting \\
\hline Sales Brain (EUA) & $\begin{array}{l}\text { En su equipo tienen la figura denominada neuromovie producer, encargada de crear vídeos. En sus servicios dispo- } \\
\text { nen de neuromovie para mensajes audiovisuales. }\end{array}$ \\
\hline Sand Research (EUA) & $\begin{array}{l}\text { Tiene una gran base de datos con más de } 500 \text { episodios de televisión, trailers y anuncios. Algunos de sus servicios } \\
\text { están destinados a valorar storyboards, animatics y hasta episodios de televisión. }\end{array}$ \\
\hline Media Science (EUA) & $\begin{array}{l}\text { Clientes: Nickelodeon, ESPN, A+E Networks, Hulu, ABC. Su fundador, Duane Varan, es conocido por crear el proyecto } \\
\text { Beyond:30 logrando reunir a grandes empresas de la industria del entretenimiento para afrontar los retos del sector } \\
\text { mediante técnicas neurocientíficas. }\end{array}$ \\
\hline Ofactor (EUA) & $\begin{array}{l}\text { Trabaja fundamentalmente en la medición de los niveles de oxitocina. La compañía ha desarrollado el sistema ZEST } \\
\text { (Zak engagement statistic) para estudiar shows de TV y películas, basado en la aplicación de la neurociencia con } \\
\text { distintos objetivos, en los que figuran el seguimiento de la audiencia y medición de la confianza y el compromiso. }\end{array}$ \\
\hline Sensum (EUA) & $\begin{array}{l}\text { Servicios a la industria cinematográfica y televisión en la edición, timing, audio y selección de escenas. Indican } \\
\text { como cliente a una broadcaster nacional. También testan videojuegos. }\end{array}$ \\
\hline BrainSigns (Italia) & $\begin{array}{l}\text { Analiza productos cinematográficos y videojuegos para mejorarlos y conocer los efectos físicos que producen. Sus } \\
\text { clientes tienen la posibilidad de realizar los tests en la Sapienza - Università di Roma. }\end{array}$ \\
\hline $\begin{array}{l}\text { Noldus Information Technology } \\
\text { (Países Bajos) }\end{array}$ & $\begin{array}{l}\text { Desarrollo y comercialización de software y hardware, y servicios de análisis de conducta animal y humana. Su } \\
\text { tecnología se emplea en laboratorios para el test de videojuegos. }\end{array}$ \\
\hline Neurosense (Reino Unido) & $\begin{array}{l}\text { Clientes: Viacom, BBC, Sky e ITV. Ofrece varias técnicas, entre las que se incluye la resonancia magnética funcional y } \\
\text { técnicas biométricas. }\end{array}$ \\
\hline Neurostrata (Reino Unido) & $\begin{array}{l}\text { Su fundador, Thom Noble, destaca por su actividad en la industria del entretenimiento. Con su anterior empresa, } \\
\text { Neuroco, trabajó con técnicas de neurociencia aplicadas a la } 20^{\text {th }} \text { Century Fox. Actualmente, está centrado en el valor } \\
\text { de la música y sonido en las industrias creativas. }\end{array}$ \\
\hline $\begin{array}{l}\text { Brain\&Research (República de } \\
\text { Corea) }\end{array}$ & Servicios a los sectores de videojuegos, cadenas de televisión (MBC, TVN o EBS), y otros. \\
\hline Buyer Brain (Rumanía) & Servicios en televisión a través del emplazamiento de productos y de anuncios en diversos contextos televisivos. \\
\hline NeuroTrend (Rusia) & Tests de emplazamiento de producto en películas, además de servicios al sector del videojuego. \\
\hline Neuro Discover (Turquía) & Está iniciando una nueva línea de estudios dedicados al cine bajo la categoría de Neuro Cinema. \\
\hline
\end{tabular}

jetivo de proponer nuevas vías y modelos para afrontar los cambios que imponen los escenarios mediáticos y el comportamiento de las audiencias (Audience Research Labs, 2011).

En España las neurociencias comienzan a encontrar su sitio, tanto en el campo de la publicidad (Andreu-Sánchez; Contreras-Gracia; Martín-Pascual, 2014), como en el del entretenimiento. En este artículo se parte de la hipótesis de que está apareciendo en España una nueva figura profesional emergente vinculada al campo de la comunicación, el experto en neuromarketing audiovisual. Para determinar su perfil se ha llevado a cabo una investigación sobre su situación, prácticas, viabilidad, retos y proyección futura.

\section{Metodología}

El objetivo principal de este trabajo consiste en determinar el estado del neuromarketing en el sector audiovisual español. Para llevar a cabo la investigación se establece una serie de subobjetivos e hipótesis a modo de guía:

SO1. Analizar el perfil del profesional del neuromarke- ting: viabilidad de la profesión, formación requerida y protocolos necesarios.

SO2. Dibujar la situación actual de este profesional en el sector audiovisual nacional: prácticas habituales, porcentaje de facturación, evolución de la cuota de mercado e impacto de los servicios de neuromarketing en el sector del entretenimiento.

SO3. Identificar las empresas del sector del entretenimiento (cadenas de televisión y productoras) que emplean estos servicios, así como conocer su visión ante el fenómeno del neuromarketing: tipos de clientes, motivaciones y necesidades del sector, beneficios y limitaciones de estas técnicas para la industria.

SO4. Valorar la proyección futura esperada del profesional del neuromarketing en el sector del entretenimiento.

Las hipótesis dimanantes del marco teórico, consecuencia de la revisión de la literatura, contrastadas en la investigación, se enumeran a continuación: 
H1. Las neurociencias se pueden aplicar al estudio del consumidor audiovisual.

H2. Los equipos necesarios para llevar a cabo investigación neurocientífica han de ser multidisciplinares.

H3. La introducción de las neurociencias en la medición de productos audiovisuales españoles es incipiente.

H4. El neuromarketing tiene carácter emergente en España, si bien débil, a través de las principales cadenas de televisión privadas nacionales, motivadas por su modelo de negocio y estructura económica.

H5. Las productoras cinematográficas y de televisión interesadas en la investigación neurocientífica no se muestran dispuestas a invertir en ella, principalmente por la falta de recursos económicos

H6. La evolución de la profesión será positiva en los próximos años.

Se ha aplicado una metodología cualitativa, concretamente entrevistas en profundidad con preguntas abiertas. Se han efectuado 18 entrevistas personales estructuradas de carácter exploratorio y prospectivo a un panel multidisciplinar, una de ellas vía telefónica. Los expertos se han clasificado así:

- empresas consultoras de neuromarketing (5);

- representantes del sector audiovisual (5);

- neurocientíficos (4);

- académicos con líneas de investigación en neuromarketing (4).

El cuestionario diseñado se ha estructurado con preguntas comunes a todos los grupos de interés y se ha completado con cuestiones propias para cada grupo. Las preguntas giran en torno a los subobjetivos de la investigación. Las entrevistas han cesado al encontrar saturación en las respuestas dentro de cada grupo y se han llevado a cabo entre junio y septiembre de 2015. Todas han sido grabadas y transcritas a excepción de una. La media de las mismas es de 70 minutos.

Dado que inicialmente se desconocían las empresas consultoras de neuromarketing que conformaban la población de interés, se realizó una investigación previa en la Red para delimitar y establecer a partir de ella la muestra objeto de estudio. Tras una búsqueda exhaustiva se encontró un total de 13 empresas que manifiestan ofrecer servicios de neuromarketing. De dicha lista se han eliminado 8 que por su especialización no eran susceptibles de ofrecer servicios de neuromarketing en el campo de la comunicación. La muestra resultante se recoge y describe en la tabla 2.

\section{Se han realizado entrevistas personales a representantes del sector audiovisual, consultoras, neurocientíficos y académi- cos investigadores en neuromarketing}

Los entrevistados ratificaron que estas 5 compañías conformaban el mercado de las consultoras de neuromarketing del país. A partir de las primeras entrevistas se añadieron a la muestra personas y compañías de interés. Para la selección de representantes del sector audiovisual se ha abordado a dos tipos de empresas:

- las que emplean técnicas neurocientíficas en sus investigaciones de mercado;

- las que aún no haciéndolo, muestran interés por las mismas o permiten realizar una radiografía sobre la investigación en el sector audiovisual.

Concretamente se contó con la colaboración de los directores de marketing del grupo Atresmedia y Mediaset España, Javier Andrés-Ortega y Javier L. Cuenllas, respectivamente, y una productora de cine de animación, junto con dos profesionales del sector televisivo que accedieron a participar de forma anónima.

Por otra parte, se realizaron entrevistas a neurocientíficos para valorar la viabilidad de la profesión y las posibilidades que ofrecen estas técnicas al estudio del sector del entretenimiento. Para ello se recurrió a investigadores de la Universidad de A Coruña (Javier Cudeiro-Mazaira y Xurxo Mariño-Alfonso), y de la Universidad de Vigo (José-Antonio Lamas-Castro y Antonio Reboreda-Prieto). Se
Mediaset ha apostado por la aplicación de la tecnología Sociograph para evaluar sus productos de ficción, ya que esta nueva metodologia de estudios de neuromarketing grupales aporta un valor añadido a los tradicionales métodos de investigación.

http://www.sociograph.es/mediaset-apuesta-por-sociograph-herramienta-de-neuromarketing-para-testar-ficcion 
contactó con otros académicos con líneas de trabajo vinculadas al neuromarketing, dentro y fuera del sector del entretenimiento, pues podían ofrecer una visión complementaria sobre el tema de interés. Con este objeto se entrevistó a María-Luisa García-Guardia y Jesús Timoteo-Álvarez, de la Universidad Complutense de Madrid, a Celia Andreu-Sánchez de la Universitat Autònoma de Barcelona y a Asunción Beerli-Palacio de la Universidad de Las Palmas.

La neurociencia como método complementario a las técnicas tradicionales busca mejorar la competitividad de las compañías mediáticas incrementando el retorno de la inversión

\section{Resultados}

De acuerdo con los científicos entrevistados, es unánime la idea de la factibilidad del estudio del consumidor audiovisual a través de técnicas neurocientíficas. Si bien este tipo de investigación es posible, los científicos apuntan a una serie de limitaciones a tener en cuenta. Las principales preocupaciones son:

- Ética: existe una preocupación latente sobre la tendencia de la neuromoda. Se alude a la praxis empresarial y a la implicación que los estudios puedan tener sobre la población.

- Interpretación de los datos: este tipo de investigación sólo podrá ofrecer ventajas competitivas si se es capaz de implementar los resultados obtenidos y, por el momento, la neurociencia tiene poca capacidad prescriptiva por sí sola sobre el comportamiento humano. La figura del psicólogo jugaría un papel relevante en este sentido.

- El tamaño de la muestra se vincula con la dificultad de acceder a una de tipo aleatoria voluntaria, representativa de la población, y la extrapolación de los resultados a la misma.
Si bien se confirma la hipótesis $\mathrm{H} 1$, este tipo de investigación no está exenta de limitaciones, superables gracias a la mejora de la tecnología no invasiva y del conocimiento científico.

La investigación de mercados con metodología neurocientífica requiere del trabajo de especialistas multidisciplinares. El equipo ha de estar compuesto por personal con formación académica en el campo de la neurociencia (biólogos, médicos, psicólogos...), además de por profesionales expertos en marketing. También se considera útil la incorporación de ingenieros biomédicos.

La necesaria especialización y experiencia en campos tan diferentes como la neurociencia y el marketing impide la existencia de un experto integral, siendo poco acertado hablar del profesional del neuromarketing en sí mismo. Todas las consultoras del estudio manifiestan disponer de equipos formados por expertos en el campo de la neurociencia (en sus distintas especializaciones), además de en marketing y comunicación. Para realizar este tipo de investigación se estima imprescindible regirse por el protocolo del método científico:

- formulación de las preguntas correctas;

- generación de hipótesis;

- diseño del experimento;

- recopilación de datos;

- interpretación de los mismos.

La metodología se determinará siempre en función de los objetivos. Puede corroborarse la hipótesis $\mathrm{H} 2$; es decir, que los equipos necesarios para realizar investigación neurocientífica han de ser multidisciplinares.

La investigación de mercados con técnicas neurocientíficas es un fenómeno incipiente, de acuerdo con todos los entrevistados que se han pronunciado al respecto. En el ámbito académico esta situación se debe al desconocimiento y la baja transferencia universidad-empresa. Las principales razones aportadas se resumen a continuación, indicando el porcentaje de consultoras que las apoyan:

Tabla 2. Consultoras de neuromarketing entrevistadas que operan en España

\begin{tabular}{|c|c|c|c|}
\hline Empresa & Responsables & Actividad & Tecnología \\
\hline BitBrain & $\begin{array}{l}\text { María López (CEO) } \\
\text { y Javier Mínguez } \\
\text { (CTO\&CSO) }\end{array}$ & $\begin{array}{l}\text { Especializada en neurotecnología. Nació como } \\
\text { spin-off de la Universidad de Zaragoza. }\end{array}$ & $\begin{array}{l}\text { Biosensores } \\
\text { Electroencefalografía (EEG) } \\
\text { Eye tracking } \\
\text { Dedicada a la ingeniería biomédica y neurotec- } \\
\text { nología, comercializa paquetes tecnológicos bajo } \\
\text { la marca Ussens (softwares que permiten analizar } \\
\text { los datos obtenidos por sus hardwares: anillos y } \\
\text { diademas). }\end{array}$ \\
\hline $\begin{array}{l}\text { Brain House } \\
\text { Institute }\end{array}$ & $\begin{array}{l}\text { Antonio Ruiz, (execu- } \\
\text { tive manager) }\end{array}$ & $\begin{array}{l}\text { Entre sus clientes se encuentra el grupo mediático } \\
\text { Atresmedia. }\end{array}$ & $\begin{array}{l}\text { Electroencefalografía (EEG) } \\
\text { Eye tracking } \\
\text { Sensores biométricos }\end{array}$ \\
\hline Inside Brain & $\begin{array}{l}\text { Francisco Arribas } \\
\text { Aguilar (director } \\
\text { general) }\end{array}$ & $\begin{array}{l}\text { Centro de neurociencia aplicada. Entre sus activi- } \\
\text { dades resaltan, en el campo publicitario, el test de } \\
\text { productos y personal y neuroweb. }\end{array}$ & $\begin{array}{l}\text { Resonancia magnética funcional } \\
\text { EEG y EEG Seco } \\
\text { Eye tracking } \\
\text { Sensores biométricos } \\
\text { Sistemas de reconocimiento facial }\end{array}$ \\
\hline Neurologyca & Juan Graña (CEO) & $\begin{array}{l}\text { Ofrecen servicios en publicidad, puntos de venta, } \\
\text { packaging. }\end{array}$ & $\begin{array}{l}\text { Electroencefalografía (EEG) } \\
\text { Sensores biométricos } \\
\text { Eye tracking }\end{array}$ \\
\hline Sociograph & $\begin{array}{l}\text { Elena Martín-Guerra } \\
\text { (directora y cofunda- } \\
\text { dora) }\end{array}$ & $\begin{array}{l}\text { Entre sus clientes se encuentra Mediaset España. } \\
\text { Además ofrecen servicios en política, coaching y } \\
\text { producto. }\end{array}$ & $\begin{array}{l}\text { Tecnología propia patentada para medir el impacto } \\
\text { de un estímulo mediante el estudio de la actividad } \\
\text { electrodérmica (EDA). }\end{array}$ \\
\hline
\end{tabular}


- El desconocimiento del neuromarketing es la principal causa aludida (80\%).

- Desconfianza y miedo: el desconocimiento por parte de los clientes potenciales eleva la desconfianza y el miedo a probar (80\%).

- Mala praxis: la dudosa actuación de terceras empresas daña la imagen de este tipo de investigación, generando frustración entre los clientes actuales y desconfianza entre los potenciales (40\%).

- Falta de actitud innovadora: tradicionalmente España no es un país con espíritu innovador. La crisis de los últimos años ha favorecido el inmovilismo entre las empresas por temor a destinar recursos a innovar y equivocarse (60\%).

- Precio: a pesar de que las consultoras sostienen mantener precios razonables, se sugiere que parte del sector audiovisual no está dispuesto a invertir en este tipo de investigación, fundamentalmente a causa de la situación financiera y laboral (falta de intermediarios) actual (20\%).

- No se ofrecen resultados con aplicabilidad a la industria audiovisual (20\%).

Todas las consultoras entrevistadas afirman haber realizado algún estudio para la industria audiovisual. El volumen de trabajo prestado al sector es desigual. Según los datos facilitados, cabe pensar que existe una empresa especializada en este sector. De manera global la media del volumen de trabajo a clientes de la industria se sitúa en un $33 \%$. En facturación la media se encuentra en un $38 \%$. Si bien es cierto que existe poca discrepancia entre volumen de trabajo y facturación, dos consultoras acusan una mayor diferencia porcentual debido al tiempo de dedicación de estos productos por su metraje.

Respecto a la evolución de la cuota de mercado en el último año, dos consultoras han aumentado sus servicios al sector audiovisual una media del $40 \%$, una tercera sostiene que se han triplicado las expectativas, y las dos restantes no han facilitado información. Los servicios a la industria audiovisual empezaron hace aproximadamente dos años y han crecido exponencialmente en el último año. No obstante, hay consenso en que todavía su aparición en el panorama general es muy incipiente y se concentra en muy pocos clientes. Por consiguiente, cabe confirmar la hipótesis H3: la introducción de las neurociencias en la medición de productos audiovisuales españoles es incipiente.

Los servicios que las consultoras ofrecen a la industria del entretenimiento apuntan a dos vías:

- análisis de contenido audiovisual;

- servicios para la mejora de la eficiencia del bloque publicitario.

Es unánime entre consultoras y académicos la idea de que estas técnicas permiten adoptar decisiones más acertadas en lo relativo a las producciones, con el correspondiente ahorro de dinero y eficiencia en la inversión. Las motivaciones de las consultoras para incorporar estas técnicas al sector audiovisual se enumeran a continuación:

- aportan información objetiva frente a otras metodologías (80\%);

- aumentan el porcentaje de eficacia/calidad de los productos para que lleguen mejor al público (80\%);

- permiten el retorno de la inversión (60\%);

- proveen criterios objetivos para la compra de contenidos audiovisuales (40\%);

- permiten prever el nivel de éxito o fracaso de las producciones desarrolladas y compradas (40\%);

- revelan el feedback de los consumidores (20\%);

- se prueban por curiosidad (20\%).

Los datos permiten concluir que la aparición del servicio de consultoría con metodología neurocientífica se circunscribe principalmente a los dos mayores grupos mediáticos privados españoles: Atresmedia y Mediaset España. Tradicionalmente la investigación de mercados en las cadenas de televisión se basaba en metodologías cuantitativas (Kantar Media) y cualitativas (focus groups), y ninguna está exenta de limitaciones. La cuantitativa no ofrece todas las explicaciones necesarias y la cualitativa presenta sesgos evidentes en las respuestas de los participantes del estudio. El neuromarketing audiovisual ofrece a las cadenas la posibilidad de obtener información objetiva sobre sus espectadores.

El grupo Mediaset España y su comercializadora Publiespaña están involucrados en estudios de contenido de ficción y bloques publicitarios respectivamente. En los últimos años el grupo ha implantado una nueva forma de producir
Welcome to the homepage of the international Neuromarketing Science \& Business Association (NMSBA). Neuromarketing can be best defined as any marketing or market by the findings or insights of brain science.

We are delighted to answer all vour questions regarding this new and exciting field. Feel free to sign up to our weekly email newsletter.

http://www.nmsba.com
Join the NMSBA!

Click here to review pricing and plans 
contenido de ficción. Dentro de los cambios estructurales abordados, la investigación neurocientífica forma parte del proceso. Para ello recurren a la contratación de una consultoría y por el momento la ficción en prime time es el único producto testado. Sus estudios emplean una pulsera que registra la actividad electrodérmica y se complementan con encuestas. Asimismo, ha de señalarse que hay distintas interpretaciones conceptuales en torno al término neuromarketing, y si bien se entiende necesariamente vinculado al registro de las respuestas cerebrales, también es utilizado para investigaciones donde se registra exclusivamente la actividad epidérmica de los sujetos. La muestra es de 90-100 personas. Los estudios se realizan simultáneamente a grupos de 30 personas adultas y jóvenes (no se testan mayores de 60 años) y con una representación mayor de mujeres que de hombres. Esta investigación se lleva a cabo en estados iniciales de grabación, contribuyendo a fundamentar y ratificar decisiones del departamento de contenido en la incorporación de cambios durante la producción. Sus estudios permiten obtener previsiones sobre el éxito o fracaso de las series. La experiencia está siendo positiva, y se prevé que en el futuro se testen programas de entretenimiento, sin descartar el cine.

Los servicios a la industria audiovisual en el último año han crecido exponencialmente y se concentran en muy pocos clientes

El grupo Atresmedia ha empleado este tipo de investigación mediante proveedores externos para testar bloques publicitarios y contenidos (programas de entretenimiento). Después de una primera iniciativa para el programa ¡Boom! se ha dejado de lado la línea de contenido, al no ser posible identificar con precisión las causas de los resultados de la investigación. Por el contrario la investigación en el área de publicidad continúa. Se trabaja principalmente sobre las variables de atención y memoria de los espectadores en el bloque comercial. En sus estudios emplean EEG (electroencefalografía), EMG (electromiograma), conductancia galvánica y actividad cardíaca. Se complementan con conocimientos de psicología cognitiva y economía del comportamiento. Estos estudios proporcionan al grupo un plus en la eficacia publicitaria, un discurso de venta innovador e incrementan la imagen de compromiso que la cadena mantiene con sus anunciantes. En cuanto a su futuro, se cree que será un apoyo más a la hora de perfilar contenidos o generar nuevas ideas. Sin embargo, se cree que no será una técnica fundamental para el negocio ni que llegue a generar más cuota.

Finalmente cabe hacer referencia a TVE, que no participó en este estudio. Conforme a lo expresado por su antiguo proveedor de investigación en neuromarketing, se sabe que aplicó algunas de las técnicas mencionadas al análisis de contenidos, aunque abandonó esa línea de trabajo por motivos económicos.

Los resultados anteriores apoyan la hipótesis $\mathrm{H} 4$, según la cual el neuromarketing en España tiene carácter emergen- te, débil, a través de las principales cadenas de televisión privadas nacionales, motivadas por su modelo de negocio y estructura económica.

La necesaria especialización y experiencia de los equipos en campos tan diferentes como la neurociencia y el marketing impide hablar actualmente de expertos integrales

Instinto y oficio son las principales armas de productores de televisión y cine. Tradicionalmente, las productoras no emplean grandes recursos a estudios de mercados, ya que no hay cultura de investigación. La decisión final de que un producto televisivo salga a la luz depende de los canales y compañías sobre las que recaen los estudios. La tendencia está cambiando, pues las grandes productoras de televisión, así como las de cine, se interesan ya por el neuromarketing audiovisual. Es generalizada la idea de la introducción de sesgos con metodologías tradicionales. A las productoras de cine les interesa evitar el sesgo de la racionalización de la respuesta de los espectadores y saber si están dentro o fuera de la película. Les interesa conocer en definitiva el engagement de sus productos. Se cree que las técnicas neurocientíficas pueden mejorar ciertos aspectos de la película y con ello contribuir a su calidad, pero en ningún caso servirán para arreglar grandes problemas de la producción. Idealmente los estudios se incorporarían en las fases intermedias de la producción; es decir, en un primer corte de la película o incluso en un animatic (versión preliminar) para las películas de animación. Un uso de interés para los productores cinematográficos consiste en conocer cómo funciona la película entre audiencias extranjeras, sobre todo para aquellos productos fácilmente exportables como es la animación. A pesar del interés, las productoras cinematográficas todavía no han dado el paso a la investigación. Los bajos presupuestos que se manejan impiden destinar fondos a investigar prototipos, a lo que se añade la falta de personal dentro de las producciones que pueda ejercer de intermediario con la consultora. Se confirma así la hipótesis H5, las productoras cinematográficas y de televisión interesadas en la investigación neurocientífica no se muestran dispuestas a invertir en ella, principalmente por la falta de recursos económicos.

La tendencia futura de la investigación de mercados con métodos neurocientíficos en el sector audiovisual se espera muy favorable por parte de los consultores. Tanto profesionales como neurocientíficos sugieren que la introducción, normalización y democratización de este tipo de investigación dependerá de:

- desarrollo tecnológico;

- estandarización tecnológica;

- mejora de la interpretación de los datos;

- avance en el conocimiento;

- abaratamiento de los estudios;

- demostración de la eficacia de la propia metodología en términos de retorno de la inversión.

En el entorno académico se prevé una tendencia positiva 
aunque en menor medida que la prevista por las consultoras, pues se alude a formas alternativas de investigación más económicas como pueden ser los big data.

En lo que respecta al futuro de la profesión, se cree que aquellas consultoras que no ofrezcan interpretaciones de valor para sus clientes desaparecerán. Una de las empresas prevé la desaparición a largo plazo de las consultoras a medida que las técnicas sean incorporadas en las compañías y se adopten como una metodología más. Igualmente se cree que es el momento óptimo de entrar en la investigación aplicada mediante el fomento de la transferencia universidad-empresa. Se cumple la hipótesis H6, la tendencia se espera que sea positiva en los próximos años.

Hasta el momento, los estudios realizados con base en el neuromarketing no han logrado satisfacer todas las necesidades de los principales grupos mediáticos españoles

\section{Discusión y conclusiones}

Las emociones constituyen la materia prima del sector audiovisual, además de tener un relevante papel en la toma de decisiones, de interés para la neurociencia. Tradicionalmente los neurocientíficos han permanecido al margen de los intereses empresariales; sin embargo, la transferencia universidad-empresa está creciendo. Una parte de la comunidad científica entrevistada afirma que en el futuro los neurocientíficos serán cada vez más demandados creándose nuevos empleos en el área de la investigación de mercados. En esta línea se apunta a la oferta formativa de master existente en Europa con dicha intención, formar científicos para abrir y cubrir futuros puestos de trabajo.

El incremento de la competencia y saturación televisiva propicia la competitividad de las cadenas y ello pasa por conocer mejor a los consumidores. La investigación de mercados con metodologías neurocientíficas aplicadas al sector audiovisual tiene carácter emergente en España, aunque su implantación definitiva se supeditará a la demostración de su eficacia.

Las principales limitaciones del neuromarketing audiovisual se relacionan con la tecnología y la interpretación de los datos, elementos que se prevén superables en los próximos años. Las productoras de cine y televisión muestran un interés creciente por el neuromarketing audiovisual aunque cabe señalar que hay varias barreras a las que se enfrentan en su implementación como el precio o la falta de cultura en investigación de mercados. Para su afianzamiento es necesario avanzar en el conocimiento de las demandas propias del sector audiovisual. Actualmente los estudios realizados con base en el neuromarketing no han logrado satisfacer todas las preguntas que los principales grupos mediáticos en España plantean. Atresmedia y Mediaset España, así como sus respectivas consultoras, se encuentran en una fase de aprendizaje mutuo. Los retos futuros a los que se enfrenta el neuromarketing audiovisual se vinculan al avance en la interpretación de datos, así como a la mejora de las técnicas de monitorización electrodérmica en el registro de la variable emoción.

\section{Bibliografía}

Andreu-Sánchez, Celia; Contreras-Gracia, Aroa; MartínPascual, Miguel-Ángel (2014). "Situación del neuromarketing en España". El profesional de la información, v. 23, n. 2, pp. 151-157.

http://dx.doi.org/10.3145/epi.2014.mar.07

Ariely, Dan; Berns, Gregory S. (2010) “Neuromarketing: the hope and hype of neuroimagining in business". Nature reviews. Neuroscience, v. 11, n. 4, pp. 284-292.

http://www.ncbi.nlm.nih.gov/pmc/articles/PMC2875927 http://dx.doi.org/10.1038/nrn2795

Audience Research Labs (2011). Beyond:30. Advertising models for a changing media landscape (third wave). Murdoch: Audience Research Lab, Murdoch University.

http://www.beyond30.org/wp-content/uploads/b30_ prospectus_2011.pdf

Babu, S. Samuel; Vidyasagar, Thalluri-Prasanth (2012). "Neuromarketing: is Campbell in soup?". The IUP journal of marketing management, v. 11, n. 2, pp. 76-100.

http://www.iupindia.in/1205/Marketing\%20Management/ Neuromarketing.html

Braidot, Néstor (2009). Neuromarketing. Barcelona: Gestión 2000. ISBN: 9788498750447

Hammou, Khalid-Ait; Galib, Hasan; Melloul, Jihane (2013) "The contributions of neuromarketing in marketing research". Journal of management research, v. 5, n. 4, pp. 20-33. http://dx.doi.org/10.5296/jmr.v5i4.4023

Hubert, Mirja; Kenning, Peter (2008). "A current overview of consumer neuroscience". Journal of consumer behaviour, v. 7, n. 4-5, pp. 272-292.

http://dx.doi.org/10.1002/cb.251

Lee, Nick; Broderick, Amanda J.; Chamberlain, Laura (2007). "What is 'neuromarketing'? A discussion and agenda for future research". International journal of psychophysiology, v. 63, n. 2, pp. 199-204. http://dx.doi.org/10.1016/j.ijpsycho.2006.03.007

Mullen, Melissa; Noble, Thom (2007). "Neuroscience: a new means of understanding". Admap, March, n. 481, pp. 39-41.

Pop, Nicolae Al.; Dabija, Dan-Cristian; Iorga, Ana-Maria (2014). "Ethical responsibility of neuromarketing companies in harnessing the market research. A global exploratory approach". Amfiteatru economic, v. 16, n. 35, pp. 26-40. http://www.amfiteatrueconomic.ro/temp/Article_1249.pdf

Tiltman, David (2005). "Market research: Mind reading". Brand republic, 23 noviembre.

http://www.brandrepublic.com/bulletins/marketresearch/ article/529442/market-research-mind-reading

Treutler, Theresa; Levine, Brian; Marci, Carl D. (2010). "Biometrics and multi-platform messaging: The medium matters". Journal of advertising research, v. 50, n. 3, pp. 243-249. http://dx.doi.org/10.2501/S0021849910091415 